\title{
Caracterização dos problemas relacionados aos pés de idosos de uma comunidade em município do interior do Estado de São Paulo
}

\author{
Characterization of feet problems in community elderly in a city of São Paulo state, Brazil
}

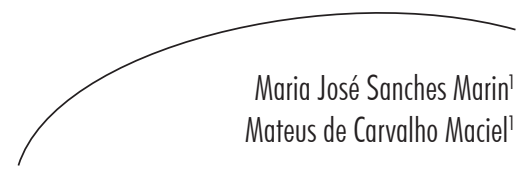

\section{Resumo}

Os problemas com os pés dos idosos, embora frequentes, são pouco reconhecidos e valorizados como uma condição que requer cuidados adequados. Este estudo teve como objetivo verificar as características dos problemas com os pés de idosos de uma comunidade. Trata-se de estudo descritivo realizado com 50 idosos pertencentes à área de abrangência de uma Unidade de Saúde da Família. Foi aplicado um instrumento que avalia condições gerais de saúde e específicas relacionadas aos pés. Constatou-se que a maioria era do gênero feminino e pertencente às classes socioeconômicas consideradas pobre e muito pobre. Os problemas de saúde mais frequentes foram a acuidade visual diminuída, hipertensão arterial sistêmica e dor em diferentes regiões do corpo. $\mathrm{Na}$ avaliação dos pés, constatam-se alterações nos pulsos; unhas compridas, deformadas e grossas; pele seca e com hiperqueratose; diminuição da sensibilidade dolorosa e vibratória. Além disso, $70 \%$ dos idosos referiram que as dores nos pés limitavam as atividades cotidianas. Depreende-se que tais situações, embora se trate de uma condição específica, revelam a intensidade dos problemas que envolvem essa parcela da população e a importância de uma atenção mais pormenorizada, o que demanda investimentos na formação de profissionais de saúde e desenvolvimento de ações interdisciplinares, com novos olhares e pressupostos de atendimento, tendo como foco a promoção da saúde.

\section{Abstract}

Problems with the feet of the elderly, though frequent, are poorly recognized and valued as a condition that requires proper care. This study aimed to determine the characteristics of the problems with the feet of community elderly. This is a descriptive study on 50 elderly belonging to the catchment area of a Family Health Unit. An instrument that assesses general health and specific conditions related to foot was applied. It was found that most were female and belonged to socioeconomic classes considered poor and very poor. The most frequent health problems were decreased visual acuity, hypertension and pain in different body regions. In the evaluation of the feet,

Palavras-chave: Saúde do Idoso. Promoção da Saúde. Pé. Deformidades do Pé.

Key words: Health of the Elderly. Health Promotion. Foot. Foot Deformities.

Curso de Enfermagem, Faculdade de Medicina de Marília. Marília, SP, Brasil.

Apoio financeiro: Fundação de Amparo à Pesquisa do Estado de São Paulo, por meio da concessão de bolsa para projeto de iniciação científica, parecer no 2012/04252-1.

Correspondência / Correspondence

Maria José Sanches Marin

E-mail:marnadia@terra.com.br 
they find themselves with alterations in the wrists; long, thick and deformed nails; dry skin and hyperkeratosis; reduced pain and vibratory sensitivity. Furthermore, $70 \%$ of seniors reported that foot pain limited daily activities. It appeared that such situations, even if it is a specific condition, reveal the intensity of the problems involving this population and the importance of further attention, which requires investment in training of health professionals and the development of interdisciplinary actions with new perspectives and assumptions of care, focusing on health promotion.

\section{INTRODUÇÃo}

No Brasil, a saúde do idoso aparece como uma das prioridades das políticas públicas, especialmente no que se refere à manutenção da autonomia e independência. As alterações funcionais desempenham importante papel nesta condição. Mesmo assim, muitos problemas apresentados por eles são pouco valorizados tanto pelos profissionais de saúde como pelo próprio idoso. Como exemplo, citam-se as alterações nos pés.

Na literatura nacional, são poucos os estudos que apontam os aspectos que contribuem para o cuidado com os pés do idoso como um requisito de preservação de sua qualidade de vida. Em estudo que identificou relação entre alterações dos pés dos idosos com os aspectos cinético-funcionais e quedas, destaca-se que tais alterações são potencialmente modificáveis por meio de prevenção, tratamento e reabilitação. ${ }^{1}$

Os problemas dos pés dos idosos estão relacionados com a deterioração da habilidade funcional e interferem na mobilidade, que é vital para manter a saúde, a funcionalidade e a independência. ${ }^{2,3}$ Mesmo tendo grande impacto na saúde e qualidade de vida, a procura por tratamento específico é baixa. ${ }^{4}$

Pacientes com problemas nos pés são mais propensos a relatar dores nas costas, quadris, joelhos e mãos, que estão também associados à diminuição do equilíbrio e ao aumento do risco de quedas. ${ }^{5}$ Presentes em aproximadamente $35 \%$ dos idosos, eles são provenientes de enfermidades, desgastes, uso de calçado inapropriado, mudanças tróficas por insuficiência vascular e problema com as unhas. ${ }^{6}$
$\mathrm{O}$ processo de envelhecimento leva à deterioração dos pés tanto do ponto de vista morfológico como funcional e envolve alterações das unhas, pele, nervos, vasos e estruturas ósseas, o que pode ser acelerado por processos patológicos. $^{7}$

Os cuidados com os pés aumentam o nível de atividade e, consequentemente, diminuem a dor e a rigidez articular associada às doenças degenerativas da articulação e melhoram o controle glicêmico, ${ }^{8}$ sendo aspecto de grande importância para a promoção da saúde do idoso.' Considerando a importância da manutenção da atividade para a promoção do envelhecimento, são relevantes os investimentos que permitam compreender e intervir nos problemas que os idosos possam apresentar com vistas a sua melhoria.

Dada a relevância dos problemas com os pés, cabe aos profissionais da atenção primária dar maior atenção aos mesmos, visto que se trata do primeiro contato dos idosos com o sistema de saúde e, muitas vezes, o único. ${ }^{10}$

Ao se atuar numa Unidade de Saúde da Família, que se pauta na promoção da saúde e que proporciona maior proximidade e vínculo com a população, constata-se a grande demanda de atendimentos de pessoas idosas devido à vulnerabilidade que as envolve, tanto do ponto de vista social como físico e psicológico.

Nesse contexto, ao ser estimulada a participação dessas pessoas nos grupos de atividade física (ginástica, alongamento, caminhada), constatou-se alta prevalência de queixas referentes a problemas nos pés, que impediam a realização dessas atividades, mesmo que existisse o desejo de realizá-las. Além disso, não se observaram intervenções relacionadas a tais problemas. 
Frente ao exposto, o propósito do estudo foi investigar as características de problemas com os pés das pessoas idosas de uma comunidade.

\section{MÉTODO}

Trata-se de estudo descritivo que permite identificar a existência de necessidades e proporcionar dados básicos para futuros estudos ou ações. ${ }^{11}$ Foi realizado numa Unidade de Saúde da Família de um município do interior paulista, com população de aproximadamente 220.000 habitantes. A unidade contava com 1.012 famílias cadastradas, num total de 4.256 pessoas, sendo que, destas, 461 se encontravam na faixa etária igual ou acima dos 60 anos de idade.

A população de referência foi constituída pelo universo de idosos residentes na área citada $(n=461)$. Os idosos foram abordados na própria unidade de saúde. Foram excluídos do estudo aqueles que não tinham condições de responder de forma coerente ao instrumento de coleta e aqueles com dependência moderada ou severa para as atividades de vida diária. Para considerar o grau de dependência, pautou-se na escala de classificação do grau de dependência, composta de 12 indicadores. Essa escala considera as atividades instrumentais de vida diária (fazer compras, pegar ônibus, administrar as finanças e tomar os medicamentos adequadamente) e as atividades básicas de vida diária (continência, vestir-se, tomar banho, comer, pentear-se, ir ao banheiro, passar para cama e sair dela), as quais são organizadas hierarquicamente, e permite classificar o idoso em "independente total", "dependente leve", "moderado" e "severo". 12

A amostra estudada compreendia 50 idosos, sendo que a coleta se encerrou no momento em que os dados se tornaram repetitivos, considerando-se a necessidade de se ter um panorama geral sobre os problemas com os pés desses idosos que vivem na comunidade.

Para a coleta de dados, foi elaborado um instrumento contendo: 1) dados sociodemográficos; 2) classe social: baseada no critério de classificação econômica do Brasil, que estima o poder de compra das pessoas, estratificando-as em classes econômicas; ${ }^{13}$ 3) Questionário Internacional de Atividade Física (IPAQ): utilizado para classificar a condição do idoso, quanto muito ativo, ativo, irregularmente ativo ou sedentário; contém perguntas em relação à frequência e à duração da realização de atividades físicas moderadas, vigorosas e da caminhada ${ }^{14}$ 4) história de saúde: são identificadas as principais alterações que acometem a saúde do idoso e que podem estar relacionadas com os problemas nos pés; 5) avaliação física dos pés, incluindo pulsos, temperatura e presença de edema; deformidade estrutural (dedos sobrepostos e retraídos); condições das unhas e da pele; sensibilidade dolorosa (utilizando um palito pontiagudo colocado em três pontos diferentes do dorso do pé e o participante, de olhos fechados, identificava a presença de sensação dolorosa), vibratória (por meio de um diapasão 128 Hertz colocado sobre a proeminência óssea do hálux bilateralmente comparado com o limiar de percepção do examinador); cinésico-postural do hálux (solicitado para o idoso fechar os olhos e apontar a posição do hálux modificada pelo examinador) e características dos calçados (adaptado aos pés e com sola antiderrapante) $;^{15}$ 6) Índice Manchester de incapacidade associada ao pé doloroso, em que se aplica um questionário com 19 questões, incluindo movimentação; a dor; preocupação com a dor e dificuldade de realizar atividades de lazer devido à dor. Em cada questão, o paciente tem de apontar a frequência dos sintomas no tempo. As respostas emitidas recebem uma pontuação: "nunca, em nenhum momento" (ponto $=0$ ), "sim, em alguns dias" (ponto=1) e "sim na maioria/todos os dias" (ponto=2). Usando-se uma estratégia de pontuação simples, somam-se todos os pontos, expressando assim o grau de incapacidade que pode variar de 0 a 38 pontos. Salienta-se que esse instrumento foi traduzido, adaptado e validado para a realidade brasileira. ${ }^{16}$ A coleta dos dados foi realizada nos meses de julho e agosto de 2012 e todos os idosos foram avaliados por um dos pesquisadores.

$\mathrm{Na}$ análise dos resultados, os dados foram digitados em planilhas do Excel 2000 for Windows para o adequado armazenamento 
das informações e, na sequência, apresentados na forma de tabelas, em frequência percentual simples, seguida de análise descritiva e comparativa com estudos realizados em outras cidades e regiões.

Para atender aos preceitos éticos de pesquisa com seres humanos, o presente estudo contou com autorização do Secretário Municipal de Saúde e com a aprovação do Comitê de Ética e Pesquisa com seres humanos da Faculdade de Medicina de Marília, sob parecer no 625/12. Os participantes foram orientados quanto ao procedimento do estudo e assinaram o Termo de Consentimento Livre e Esclarecido.

\section{RESULTADOS}

Quanto ao perfil sociodemográfico da população estudada, destaca-se que a maioria (29; 58\%), encontrava-se na faixa etária de 60 a 69 anos; era do sexo feminino (30; 60\%); vivia com companheiro (34; 68\%); pertencia à classe social D (26; $52 \%)$; e se caracterizava como ativa (26; 52\%), conforme se observa na tabela 1 .

Tabela 1. Distribuição dos participantes do estudo de acordo com a caracterização sociodemográfica. Marília-SP, 2012. $(\mathrm{n}=50)$.

\begin{tabular}{|c|c|c|}
\hline Dados de identificação & $\mathrm{n}$ & $\%$ \\
\hline \multicolumn{3}{|l|}{ Faixa etária } \\
\hline $60-69$ & 29 & 58 \\
\hline $70-79$ & 16 & 32 \\
\hline 80 ou mais & 5 & 10 \\
\hline \multicolumn{3}{|l|}{ Sexo } \\
\hline Masculino & 20 & 40 \\
\hline Feminino & 30 & 60 \\
\hline \multicolumn{3}{|l|}{ Estado marital } \\
\hline Vive com companheiro & 34 & 68 \\
\hline Vive sem companheiro & 16 & 32 \\
\hline \multicolumn{3}{|l|}{ Com quem vive } \\
\hline Companheiro(a) & 13 & 26 \\
\hline Companheiro(a)+ filhos/netos & 21 & 42 \\
\hline Irmão/filho/neto & 15 & 30 \\
\hline Sozinho (a) & 1 & 2 \\
\hline \multicolumn{3}{|l|}{ Classe social } \\
\hline B1 & 2 & 4 \\
\hline $\mathrm{C}$ & 22 & 44 \\
\hline $\mathrm{D}$ & 26 & 52 \\
\hline \multicolumn{3}{|l|}{ Atividade física } \\
\hline Muito ativo & 1 & 2 \\
\hline Ativo & 26 & 52 \\
\hline Irregularmente ativo & 15 & 30 \\
\hline Sedentário & 2 & 4 \\
\hline Não se aplica & 6 & 12 \\
\hline
\end{tabular}


Os problemas de saúde mais frequentes entre os idosos estudados referiram-se à diminuição da acuidade visual (41; 82\%), hipertensão arterial sistêmica $(39 ; 78 \%)$ e dor em diferentes partes do corpo (37; 74\%), conforme tabela 2 .

Tabela 2. Distribuição dos participantes do estudo de acordo com os problemas de saúde/hábitos. Marília-SP, 2012. ( $\mathrm{n}=50)$.

\begin{tabular}{lcc}
\hline \multicolumn{1}{c}{ Problemas de saúde/hábitos } & $\mathrm{n}$ & $\%$ \\
\hline Artrite & 20 & 40 \\
Diminuição da acuidade visual & 41 & 82 \\
HAS & 39 & 78 \\
Dor & 37 & 74 \\
Problemas circulatórios & 22 & 44 \\
Quedas & 22 & 44 \\
Diabetes & 18 & 36 \\
Dislipidemia & 14 & 28 \\
Fumo & 12 & 24 \\
Osteoporose & 12 & 24 \\
Diminuição da acuidade auditiva & 10 & 20 \\
Álcool & 7 & 14 \\
Injuria recente & 6 & 12 \\
Gota & 4 & 8 \\
\hline
\end{tabular}

$\mathrm{Na}$ tabela 3, encontram-se as alterações relacionadas aos pés, sendo as mais frequentes o pulso tibial posterior diminuído $(21 ; 42 \%)$; unhas deformadas/encurvadas $(28 ; 56 \%)$ e presença de hiperqueratose/calos (38; 76\%). Além disso, alguns tinham a sensibilidade dolorosa ausente ou diminuída $(11 ; 22 \%)$ e oito $(16 \%)$ apresentaram formato de calçado inadequado. 
Tabela 3. Distribuição dos participantes do estudo de acordo com os problemas com os pés. MaríliaSP, 2012. $(\mathrm{n}=50)$.

\begin{tabular}{|c|c|c|}
\hline Problemas com os pés & $\mathrm{n}$ & $\%$ \\
\hline \multicolumn{3}{|l|}{ Pulso/deformidades } \\
\hline Pulso tibial posterior alterado & 21 & 42 \\
\hline Edema & 18 & 36 \\
\hline Pulso pedioso & 17 & 34 \\
\hline Hálux valgo & 10 & 20 \\
\hline Retração dos dedos & 6 & 12 \\
\hline Dedos sobrepostos & 4 & 8 \\
\hline \multicolumn{3}{|l|}{ Unhas } \\
\hline Deformada/encurvada & 28 & 56 \\
\hline Grossa & 23 & 46 \\
\hline Quebradiça/trincada/lascada & 21 & 42 \\
\hline Compridas & 17 & 34 \\
\hline Descolorida & 11 & 22 \\
\hline Higiene inadequada & 8 & 16 \\
\hline Encravada & 5 & 10 \\
\hline \multicolumn{3}{|l|}{ Pele } \\
\hline Hiperqueratose /calos & 38 & 76 \\
\hline Seca & 29 & 58 \\
\hline Lesões & 15 & 30 \\
\hline Temperatura alterada & 10 & 20 \\
\hline Fissura & 8 & 16 \\
\hline Maceração & 6 & 12 \\
\hline Umidade & 4 & 8 \\
\hline Eritema & 2 & 4 \\
\hline \multicolumn{3}{|l|}{ Sensibilidade ausente } \\
\hline Dolorosa & 11 & 22 \\
\hline Vibratória & 8 & 16 \\
\hline Cinésico-postural (hálux) & 1 & 2 \\
\hline \multicolumn{3}{|l|}{ Calçado/adequação } \\
\hline Formato & 8 & 16 \\
\hline Tamanho & 5 & 10 \\
\hline
\end{tabular}


$\mathrm{Na}$ tabela 4, encontram-se distribuídos os itens que compõem o índice de Manchester, que avalia a incapacidade associada ao pé doloroso, destacando-se a grande proporção de idosos que, por causa da dor nos pés alguns ou todos os dias, tinham suas atividades de movimentação, trabalho e lazer restritas, bem como se sentiam incomodados e preocupados com essa condição.

Tabela 4. Distribuição dos participantes do estudo de acordo com a incapacidade associada ao pé doloroso no idoso. Marília-SP, 2012. ( $\mathrm{n}=50)$.

\begin{tabular}{|c|c|c|c|c|c|c|}
\hline \multirow{2}{*}{$\begin{array}{c}\text { Frequência } \\
\text { Por causa das dores nos pés }\end{array}$} & \multicolumn{2}{|c|}{ Nunca } & \multicolumn{2}{|c|}{ Alguns dias } & \multicolumn{2}{|c|}{ Todos os dias } \\
\hline & $\mathrm{n}$ & $\%$ & $\mathrm{n}$ & $\%$ & $\mathrm{n}$ & $\%$ \\
\hline Evito caminhar fora de casa & 33 & 66 & 11 & 22 & 6 & 12 \\
\hline Evito caminhar longas distâncias & 30 & 60 & 8 & 16 & 12 & 24 \\
\hline Caminho com dificuldade & 31 & 62 & 11 & 22 & 8 & 16 \\
\hline Caminho vagarosamente & 33 & 66 & 7 & 14 & 10 & 20 \\
\hline $\begin{array}{l}\text { Ao caminhar, tenho que parar e } \\
\text { descansar meus pés }\end{array}$ & 36 & 72 & 5 & 10 & 9 & 18 \\
\hline $\begin{array}{l}\text { Evito caminhar sobre superfícies ásperas } \\
\text { ou irregulares }\end{array}$ & 28 & 56 & 14 & 28 & 8 & 16 \\
\hline Evito ficar de pé por muito tempo & 27 & 54 & 11 & 22 & 12 & 24 \\
\hline $\begin{array}{l}\text { Pego ônibus, metrô ou ando de carro } \\
\text { com frequência }\end{array}$ & 30 & 60 & 14 & 28 & 6 & 12 \\
\hline $\begin{array}{l}\text { Preciso de ajuda nas tarefas domésticas/ } \\
\text { compras }\end{array}$ & 39 & 78 & 5 & 10 & 6 & 12 \\
\hline $\begin{array}{l}\text { Ainda faço tudo, mas com mais dor ou } \\
\text { desconforto }\end{array}$ & 33 & 66 & 12 & 24 & 5 & 10 \\
\hline Fico irritado quando meus pés doem & 28 & 56 & 16 & 32 & 6 & 12 \\
\hline $\begin{array}{l}\text { Estou sempre preocupado(a) com relação } \\
\text { aos meus pés }\end{array}$ & 32 & 64 & 11 & 22 & 7 & 14 \\
\hline $\begin{array}{l}\text { Preocupo-me com relação aos sapatos } \\
\text { que preciso calçar }\end{array}$ & 33 & 66 & 9 & 18 & 8 & 16 \\
\hline Tenho dores constantes nos pés & 20 & 40 & 20 & 40 & 10 & 20 \\
\hline Meus pés doem mais de manhã & 41 & 82 & 5 & 10 & 4 & 8 \\
\hline Meus pés doem mais à noite & 31 & 62 & 15 & 30 & 4 & 8 \\
\hline Sinto dores em pontadas nos meus pés & 42 & 84 & 6 & 12 & 2 & 4 \\
\hline $\begin{array}{l}\text { Sou incapaz de realizar o trabalho que } \\
\text { fazia antes }\end{array}$ & 32 & 64 & 11 & 22 & 7 & 14 \\
\hline $\begin{array}{l}\text { Não consigo mais realizar todas minhas } \\
\text { atividades anteriores }\end{array}$ & 28 & 56 & 14 & 28 & 8 & 16 \\
\hline
\end{tabular}


A pontuação individual, que pode variar de 0 (nenhum problema) a 38 (problema todos os dias em todos os itens) entre os idosos estudados, foi de 0 a 33, com uma média de 9,6. Destacase, no entanto, que $70 \%$ dos participantes do estudo manifestaram algum tipo de incômodo decorrente da dor nos pés.

\section{DISCUSSÃO}

Segundo dados do estudo, os problemas com os pés dos idosos são frequentes e com características próprias do processo de envelhecimento e das doenças a ele inerentes. A maioria dos participantes encontra-se na faixa etária entre 60 e 69 anos (55\%) e, portanto, numa fase da vida em que os investimentos na promoção da saúde são essenciais para manter sua qualidade.

Ao ser analisado o perfil motor de idosos numa cidade do interior do Rio Grande do Sul, comparando-se as faixas etárias de 60 a 70 anos e de 71 a 83 anos, foram constatados resultados superiores em idosos do grupo com menor idade. ${ }^{17}$ Por outro lado, ao comparar os gêneros masculino e feminino, observou-se que as mulheres apresentam as piores pontuações. Mesmo que no presente estudo não tenham sido analisadas as diferenças entre os gêneros, tais dados contribuem para reflexões a respeito dessa parcela da população que, embora apresente maior fragilidade nos aspectos biopsicossociais, esta se verifica em maior quantidade, o que é claramente evidenciado na literatura. ${ }^{1,4,16-18}$

Referindo-se à classe socioeconômica, os idosos estudados pertenciam, principalmente, às classes $\mathrm{C}$ e $\mathrm{D}$, ou seja, de pobres e muito pobres, respectivamente. Existe forte associação entre idosos de baixa renda com pior condição de saúde, pior função física e dificuldade de acesso aos serviços de saúde. ${ }^{19}$ Além disso, tais idosos tendem a apresentar maior volatilidade em sua estrutura de gastos, quase sempre decorrentes do inesperado surgimento de despesas, principalmente com a saúde. ${ }^{20}$
Ressalta-se, ao se considerar a pobreza entre os idosos no Brasil, que $70 \%$ dos aposentados e pensionistas do Instituto Nacional do Seguro Social (INSS) recebem um salário mínimo por mês, ${ }^{21} \mathrm{o}$ que se revela insuficiente para o atendimento satisfatório das necessidades de sobrevivência, principalmente para aqueles que envelhecem com doenças e incapacidades. ${ }^{22}$ Referindo-se especificamente aos problemas com os pés dos idosos, estudo australiano, ao concluir que esses representam grande impacto na piora da qualidade de vida e da condição de saúde referida, sugere que os idosos deveriam ter acesso regular a tratamento podológico. ${ }^{4}$ Desta forma, questiona-se: qual seria a possibilidade para a maioria dos idosos brasileiros de terem acesso a essa forma de cuidado?

A população estudada é constituída, em sua maioria, por idosos ativos ou irregularmente ativos. Resultados semelhantes foram encontrados quando comparado o nível de atividade física em diferentes faixas etárias. Com exceção do nível muito ativo, que diminui na medida em que aumenta a faixa etária, parece não existir relação entre nível de atividade física e idade cronológica. ${ }^{14} \mathrm{Na}$ verificação do nível de atividade física habitual e o tempo de prática em idosas que praticavam atividades físicas regulares, constatou-se que $82,1 \%$ praticavam 150 minutos ou mais de atividade por semana, o que as classificou como ativas. ${ }^{23}$ Considerandose a importância da manutenção da atividade física na velhice e o fato de que muitas pessoas nessa fase da vida procuram manter-se ativas, os problemas com os pés precisam ser valorizados, principalmente pelas políticas de saúde.

Os problemas de saúde referidos pelos idosos, assim como em outros estudos, também se revelam múltiplos e complexos, com destaque para a hipertensão arterial sistêmica e outros problemas circulatórios, a acuidade visual diminuída e dor em diferentes partes do corpo. A diminuição da acuidade visual, por exemplo, está associada ao impacto negativo sobre a qualidade de vida, refletindo-se na autoestima, status social e atividades ocupacionais, podendo desencadear problemas psicológicos, sociais e econômicos. ${ }^{24}$ 
Quanto à dor, ressaltou-se no questionamento sobre sua presença, que embora bastante frequente, foram poucos os que relataram dor nos pés. Porém, quando aplicado o questionário específico para avaliar este tipo de dor, 36 (72\%) disseram que tal problema repercute de alguma forma em sua vida, o que confirma a desatenção dada a isso pelo idoso. Em estudo realizado com 104 idosos que apresentavam dores nos pés, apenas $13 \%$ procuraram tratamento específico, sendo que o principal motivo pelo qual não o fizeram foi considerarem este problema como não sendo algo realmente grave. ${ }^{4}$

Entre os problemas com os pés, as alterações dos pulsos e edema, que se instalam em consequência das doenças vasculares, são consideradas a condição de maior gravidade, devido à rapidez do seu aparecimento, da imprevisibilidade da sua evolução, da impotência funcional e dos riscos de má evolução?

A presença de hálux valgo, comumente conhecido como joanete, presente em 20\% dos idosos do estudo, leva tanto à instabilidade estrutural como à postural. ${ }^{7,25} \mathrm{Na}$ correlação do pé geriátrico com requisitos cinético-funcionais de idosos que frequentavam um ambulatório de geriatria, constatou-se que todos eles apresentavam algum grau de hálux valgo, porém nenhum em grau grave. ${ }^{4}$ Foi encontrada também forte correlação entre deformidades nos pés e a perda da habilidade funcional em idosos. ${ }^{3}$

Os transtornos nas unhas também são frequentes entre os idosos e, muitas vezes, estão associados à limitação apresentada para cuidar dos pés. Aponta-se que $36 \%$ dos idosos têm dificuldade de cortar as unhas e que esse índice aumenta para $49 \%$ após a ocorrência de queda. ${ }^{26}$ Dosidosos estudados, mais de um terço apresentou unhas compridas, sendo que o estudo de revisão de literatura aponta que essa porcentagem varia de $28 \%$ a $40,4 \%,{ }^{1}$ acrescentando que, em quase um quinto deles, as unhas apresentaram-se sujas. Além disso, muitos apresentaram transtornos tróficos, como unhas quebradiças, deformadas e grossas. Tais alterações dificultam o uso de calçado apropriado, além de causar desconforto.
Alterações da pele, principalmente a hiperqueratose, foram frequentes entre os participantes do estudo. A hiperqueratose é entendida como zonas de calosidades que ocorrem em locais de proeminência óssea depois de muito tempo de pressão e atrito, podendo ocasionar dor e dificuldade para deambular, além da possibilidade de ulceração e infecção.?

Outro problema relevante encontrado na avaliação dos pés dos idosos foi a diminuição da sensibilidade dolorosa e vibratória. A perda da sensibilidade superficial do pé decorre comumente de neuropatia diabética e contribui para o surgimento de lesões. ${ }^{27}$

A intensidade e a complexidade dos problemas encontrados nos pés dos idosos se intensificam quando se observa a quantidade deles que utilizam calçados inadequados. É comum o uso do "chinelo de dedos", visto ser este um hábito desenvolvido também durante toda a vida. Além disso, alterações, como deformidades das unhas e proeminências ósseas, entre outras, impedem o uso de calçado adequado. ${ }^{28}$

Por fim, é importante salientar algumas limitações do estudo, uma vez que foram verificados os pés de idosos que vivem na comunidade e que não apresentam limitação de movimentos, os dados não podem ser generalizados, pois, possivelmente, se fossem avaliadas as mesmas condições em contextos distintos, os achados não seriam os mesmos. Além disso, embora os dados mostrem a intensidade dos problemas com os pés nos idosos avaliados, não se trata de uma amostragem probabilística, o que impede sua extrapolação.

\section{CONCLUSÃO}

Os dados do presente estudo revelam que os problemas com os pés dos idosos avaliados foram frequentes. Por suas características, identifica-se que eles interferem especialmente na limitação de movimentos, essencial para a manutenção da qualidade de vida dessas pessoas. Acrescenta-se o fato de a maioria ser de idosas, as quais comumente 
apresentam maior vulnerabilidade nas condições de saúde, e pertencerem a classes socioeconômicas pouco favorecidas, o que dificulta o acesso ao necessário atendimento à saúde, especialmente por se tratar de uma condição pouco valorizada, como é o caso dos cuidados com os pés.

Os problemas dos pés dos idosos avaliados integram uma rede de consequências oriundas do próprio processo de envelhecimento ou de múltiplas doenças, como se observa nas alterações do pulso pedioso e tibial e de sensibilidade periférica, no hálux valgo, hiperqueratose e pele seca. Outro aspecto importante confirmado no presente estudo é a evidência de que grande quantidade de idosos, devido à dor nos pés, acaba

\section{REFERÊNCIAS}

1. Aikawa AC, Paschoal SMP, Caromano FA, Fu C, Tanaka C, Ide MR. Estudo Correlacional do pé geriátrico com requisites cinético-funcionais. Fisioter Mov [Internet] 2009 [citado 05 Nov 2012];22(3):395405. Disponível em: http://www2.pucpr.br/reol/ index.php/RFM?dd1=2816\&dd99= view

2. Garrow, AP, Papageogiou AC, Silman AJ, Thomas E, Jayson MI, Macfarlane GJ. Development and validation of a questionnaire to assess disabling foot pain. Pain [Internet] 2000 [citado 05 nov 2012];85(12):107-13. Disponível em: http://www.sciencedirect. com/science/article/pii/S0304395999002638\#

3. Spink MJ, Fotoohabadi MR, Wee E, Hill KD, Lord SR, Menz HB. Foot and ankle strength, range of motion, posture, and deformity are associated with balance and functional ability in older adults. Arch Phys Med Rehabil [Internet] 2011 [citado 15 Fev 2014];92(1):68-75. Disponível em: http://www.archivespmr.org/article/S0003-9993(10)00801-4/fulltext

4. Kaoulla P, Frescos N, Menz HB. A survey of foot problems in community-dwelling older Greek Australians. J Foot Ankle Res [Internet] 2011 [citado 07 Nov 2012];4(1):23. Disponível em: http://www. jfootankleres.com/content/pdf/1757-1146-4-23.pdf

5. Menz HB, Tiedemann A, Kwan MM, Plumb K, Lord SR. Foot pain in community-dwelling older people: an evaluation of the Manchester Foot Pain and Disability Index. Rheumatology (Oxford) [Internet] 2006 [citado 07 Nov 2012];45(7):863-7. Disponível em: http://rheumatology.oxfordjournals.org/ content/45/7/863.full.pdf + html limitando muitas das atividades cotidianas, o que compromete sua autonomia e qualidade de vida.

Ressalta-se que os problemas com os pés desses idosos, embora se trate de uma condição específica, revelam a intensidade da situação que os envolve e a importância de uma atenção pormenorizada. Devido ao crescente aumento de idosos na população em geral, é importante mantê-los ativos e produtivos ao longo de suas vidas. Nesta perspectiva, torna-se evidente a necessidade de investir na formação de profissionais de saúde e desenvolver ações interdisciplinares, visando a um atendimento mais adequado, para que os idosos possam não só viver mais, mas viver melhor.

6. Robledo LMG. Alteraciones de los pies. In: Pérez EA, Galinsky D, Martinez FM, Salas AR, Ayéndez MS, editores. La atención de los ancianos: um desafio para los años noventa. Washington: Organización Panamerica de la Salud; 1997. p. 153-8. (Publicación Científica, 546)

7. Ferrari SC, Santos FC, Araújo MSL, Cendoroglo MS, Trevisani VFM. Patologias no pé do idoso. RBCEH [Internet] 2009 [citado 07 Nov 2012];6(1):106-18. Disponível em: www.upf.br/seer/index.php/rbceh/article/ download/735/485

8. Kelechi T. Foot care in the home: nursing and agency responsibilities. Home Healthc Nurse [Internet] 1996 [citado 07 Nov 2012];14(9):721-31. Disponível em: http://ovidsp.tx.ovid.com/sp-3.8.0b/ovidweb. cgi? $\mathrm{T}=\mathrm{JS} \& \mathrm{PAGE}=$ fulltext $\& \mathrm{D}={ }_{\mathrm{Ovft}} \& \mathrm{AN}=00004045$ 199609000-00010\&NEWS $=\mathrm{N} \& \mathrm{CSC}=\mathrm{Y} \& \mathrm{CHANNE}$ $\mathrm{L}=$ PubMed

9. Stolt M, Suhonen R, Puukka P, Viitanen M, Voutilainen P, Leino-Kilpi H. Foot health and selfcare activities of older people in home care. J Clin Nurs [Internet] 2012 [citado 15 Fev 2014];21(2122):3082-95. Disponível em: http://onlinelibrary. wiley.com/doi/10.1111/j.1365-2702.2012.04223.x/pdf

10. Menz HB, Jordan KP, Roddy E, Croft PR. Characteristics of primary care consultations for musculoskeletal foot and ankle problems in the UK. Rheumatology (Oxford) [Internet] 2010 [citado 15 Fev 2014];49(7):1391-8. Disponível em: http:// rheumatology.oxfordjournals.org/content/49/7/1391. full.pdf + html 
11. Trivinõs ANS. Introdução à pesquisa em ciências sociais: a pesquisa qualitativa em educação. São Paulo: Atlas; 1987.

12. Ramos LR, Perracini M, Rosa TE, Kalache A. Significance and management of disability among urban elderly residents in Brazil. J Cross Cult Gerontol [Internet] 1993 [citado 07 Nov 2012];8(4):313-23. Disponível em: http://download.springer.com/static/ pdf/139/art\%253A10.1007\%252FBF00972560.pdf?au th66=1362161151_6606ff9fc4c7edd8ade4cf2799b8c9d c\&ext $=. p d f$

13. Pereira VR. Métodos alternativos no critério Brasil para a construção de indicadores sócio-econômico: teoria da resposta ao item [dissertação na Internet]. Rio de Janeiro: Pontifícia Universidade Católica do Rio de Janeiro; 2004 [citado 07 Nov 2012]. 103 p. Disponível em: http://www.maxwell.lambda.ele.pucrio.br/acessoConteudo.php?nrseqoco $=13795$

14. Matsudo SM, Matsudo VR, Araújo T, Andrade D, Andrade E, Oliveira L, et al. Nível de atividade física da população do Estado de São Paulo: análise de acordo com o gênero, idade, nível socioeconômico, distribuição geográfica e de conhecimento. Rev Bras Ciênc Mov [Internet] 2002 [citado 07 Nov 2012];10(4):41-50. Disponível em: http://portalrevistas. ucb.br/index.php/RBCM/article/viewFile/469/495

15. Mutarelli EG. Propedêutica neurológica: do sintoma ao diagnóstico. São Paulo: Sarvier; 2000.

16. Ferrari CS, Santos FC, Guarnieri AP, Salvador N, Correa AZAH, Hala AZA, et al. Índice Manchester de incapacidade associada ao pé doloroso no idoso: tradução, adaptação cultural e validação para a língua portuguesa. Rev Bras Reumatol [Internet] 2008 [citado 10 Nov 2012];48(6):335-41. Disponível em: http://www.scielo.br/pdf/rbr/v48n6/06.pdf

17. Piccoli JCJ, Santos GA, Ferrareze ME, Junior WH. Parâmetros motores e envelhecimento: um estudo de idosos de 60 a 83 anos de Ivoti, RS. Texto \& contexto enferm [Internet] 2009 [citado 10 Nov 2012];8(2):30618. Disponível em: http://revistaseletronicas.pucrs.br/ fo/ojs/index.php/fass/article/viewFile/6346/4645

18. Marin MJS, Cecílio LCO. Necessidades de saúde de idosos de uma unidade de saúde da família. Rev Bras Geriatr Gerontol [Internet]. 2009 [citado 10 nov 2012];12(1):63-76. Disponível em: http://www.crdeunati.uerj.br/img_tse/v12n1/pdf/art_5.pdf

19. Costa MFL, Barreto S, Giatti L, Elizabeth U. Desigualdade social e saúde entre idosos brasileiros: um estudo baseado na pesquisa nacional por amostra de domicílios. Cad Saúde Pública [Internet] 2003 [citado 10 Nov 2012];19(3):745-57. Disponível em: http://www.scielo.br/pdf/csp/v19n3/15878.pdf

20. Barros RP, Mendonça R, Santos D. Incidência e natureza da pobreza entre idosos do Brasil [Internet]. Rio de Janeiro: IPEA;1999 [citado 10 Nov 2012] . (Texto para discussão; 686). 27 p. Disponível em: http://www.ipea.gov.br/pub/td/td0686.pdf

21. Goldman SN. As dimensões sociopoliticas do envelhecimento. In: Py L, Pacheco JL, Sá JLM, Goldman SN, organizadores. Tempo de envelhecer: percursos e dimensões psiocossiais. Rio de Janeiro: Nau; 2004. p. 61-81.

22. Fernandez MGM, Santos SR. Políticas públicas e direitos do idoso: desafios da agenda social do Brasil contemporâneo. Achegas.net [Internet] 2007 [citado 2012 Dez 10];(34):49-60. Disponível em: http://www. achegas.net/numero/34/idoso_34.pdf

23. Binotto MA, Borgatto AF, Farias SF. Nível de atividade física: questionário internacional de atividades físicas e tempo de prática em mulheres idosas. Rev Bras Geriatr Gerontol [Internet]. 2010 [citado $10 \mathrm{dez}$ 2012];13(3):425-34. Disponível em: http://revista.unati. uerj.br/pdf/rbgg/v13n3/v13n3a09.pdf

24. Filho VTFB, Ventura RU, Brandt CT, Sarteschi CV, Marcelo C. Impacto do déficit visual na qualidade de vida em idosos usuários do sistema único de saúde vivendo no sertão de Pernambuco. Arq Bras Oftalmol [Internet]. 2012 [citado 10 dez 2012];75(3):161-5. Disponível em: http://www.scielo.br/pdf/abo/ v75n3/02.pdf

25. Nix SE, Vicenzino BT, Smith MD. Foot pain and functional limitation in healthy adults with hallux valgus: a cross-sectional study. BMC Musculoskelet Disord [Internet] 2012 [citado 15 Fev 2014];13:1-10. Disponível em: http://www.biomedcentral.com/ content/pdf/1471-2474-13-197.pdf

26. Fabrício SCC, Rodrigues RAP. Percepção de idosos sobre alterações das atividades da vida diária após acidentes por queda. Rev Enferm UERJ [Internet]. 2006 [citado 10 dez 2012];14(4):531-7. Disponível em: http://www.facenf.uerj.br/v14n4/v14n4a07.pdf

27. Garbino JA. Avaliação eletroneuromiográfica da neuropatia diabética. In: Kuhn P. O pé diabético. São Paulo: Atheneu; 2006. p. 159-67.

28. Marin JS, Castilho NC, Myzato JM, Ribeiro PC, Candido DV. Características dos riscos para quedas entre idosos de uma unidade de saúde da família. REME Rev Min Enferm [Internet] 2007 [citado 10 Dez 2012];11(4):369-74. Disponível em: http://www. revenf.bvs.br/pdf/reme/v11n4/v11n4a04.pdf 